\title{
The effects of native and light induced defects on optoelectronic properties of hydrogenated amorphous silicon-germanium (a-SiGe:H) alloy thin films
}

\author{
M. Güneş · M. E. D. Yavas · J. Klomfass · \\ F. Finger
}

Received: 14 November 2008/Accepted: 31 March 2009/Published online: 18 April 2009

(C) Springer Science+Business Media, LLC 2009

\begin{abstract}
Effects of native and light induced defects states in hydrogenated amorphous silicon-germanium alloy thin films with different Ge concentrations have been investigated by using steady-state photoconductivity, dual beam photoconductivity (DBP), transmission spectroscopy and photothermal deflection spectroscopy (PDS) techniques. In the annealed state, sub-bandgap absorption spectra obtained from both PDS and DBP overlap very well at energies above $1.4 \mathrm{eV}$. However, differences in $\alpha$ $(\mathrm{h} v)$ spectrum exist in the lower energy part of absorption spectrum. The $\alpha(\mathrm{h} v)$ value measured at $1.0 \mathrm{eV}$ is the lowest for $10 \% \mathrm{Ge}$ sample and increases gradually as $\mathrm{Ge}$ content of the sample increases. In the light soaked state, time dependence of photoconductivity decay obeys to $t^{-x}$ power law, where $x$ changes from 0.30 to 0.60 for samples with low Ge content and 0.05-0.1 for samples with high Ge content. Correspondingly, the increase of the sub-bandgap absorption coefficient at lower energies obeys to $t^{y}$ power law, where $y$ values are lower than the $x$ value of the same sample. It can be inferred that sub-bandgap absorption and photoconductivity measurements are not controlled by the same set of defects created in the bandgap of alloys.
\end{abstract}

M. Güneş ( $₫)$

Department of Physics, Faculty of Sciences and Arts, Mugla University, Kotekli Yerleşkesi, 48000 Mugla, Turkey

e-mail: mehmet.gunes@mu.edu.tr

M. E. D. Yavas

Department of Physics, Izmir Institute of Technology, Izmir, Turkey

J. Klomfass $\cdot$ F. Finger

IEF-5 Photovoltaics, Forschungszentrum Jülich, GmbH, 52425

Jülich, Germany

\section{Introduction}

Hydrogenated amorphous silicon-germanium (a-SiGe:H) alloy thin films are potential candidates to replace the lower bandgap absorber layer in multi junction solar cells $[1,2]$. Alloying amorphous silicon with Ge results in a decreased bandgap [3-5] which beter matches solar spectrum in the bottom cell $[1,2]$. Contrarily, the density of deep defects in the bandgap increases with increased $\mathrm{Ge}$ alloying which finally degrades the lifetime of photogenerated carriers [3-5]. Even though earlier studies reported negligible Staebler-Wronski effect (SWE) in a-SiGe:H mainly due to poor material quality available at those times $[6,7]$, high quality alloy films prepared after improved deposition systems exhibited a substantial SWE under light soaking similar to that intrinsic to pure a-Si:H [8-16]. For this reason, investigations of the light induced defect creation as well as native defects in the alloy as a function of Ge content has become an important issue to be understood and resolved both scientifically and technologically. As compared to the research undertaken during the last three decades on the SWE seen in pure a-Si:H [17], only a small number of studies have been reported for the native and light induced defects in a-SiGe:H alloys [8-16]. Fundamental investigations of these defects have been carried out using electron spin resonance (ESR) [4, 5, 10], steady-state photoconductivity (SSPC) $[8,12,18]$, modulated photoconductivity (MPC) $[19,20]$, drive level capacitance profiling (DLCP) [21], and sub-bandgap absorption methods [3, 8, 18, 21-24]. None of these techniques provides a complete description of all types of defects present in the alloy due to limitations in the probing nature of the method or the sample geometry itself. However, certain direct evidences were obtained from each method for understanding of native and light induced defect states in 
a-SiGe:H. ESR clearly identifies silicon and germanium neutral dangling bond defects both in the annealed and light soaked states $[4,5,10]$. Similarlary, MPC detects a second deep defect band at energy $0.78 \mathrm{eV}$ from the conduction band [25] in addition to the characteristic single defect band observed at $0.65 \mathrm{eV}$ for pure a-Si:H [26]. However, the exact nature of these defect bands of MPC were not explicitely understood yet. In addition, the importance of charged defect states was reported already for higher Ge content alloys using MPC method [25]. Furthermore, the sub-bandgap absorption methods have been extensively used for the investigation of native and light induced defects in pure a-Si:H [3, 8, 18, 22-24]. Since the methods provide the absorption coefficient $\alpha(\mathrm{h} v)$ spectrum from visible to the sub-bandgap energies from which the optical gap $\mathrm{E}_{04}$, the slope of valence band tails $\mathrm{E}_{0 \mathrm{v}}$ representing the degree of disorder and density of deep defect states were obtained. The techniques and methodologies established for pure a-Si:H such as photothermal deflection spectroscopy (PDS) [3, 24, 27] and constant photocurrent method (CPM) [8, 13, 18, 22, 23, 27] have been directly applied to study defect sates in a-SiGe:H films. It was found that accurate determination of the subbandgap absorption $\alpha(\mathrm{h} v)$ spectrum was affected by several factors [22, 24, 28]. The effects of high density of surface states, substrate absorption in PDS spectrum, the effects of interference fringes and inaccuracies due to normalization of photoconductivity spectrum to absolute $\alpha$ (hv) spectrum independently obtained from transmission and reflection measurements [22, 24, 28] introduce significant errors in the resulting $\alpha$ (hv) spectrum. Furthermore, final derivation of the density of defect states from the accurate $\alpha(h v)$ spectrum is also limited to the calibration constant used in both PDS and CPM techniques. Those calibration constants were obtained from the ESR measurements, which only considers the neutral silicon dangling bonds as major defect states. The effects of significant charged defect states present both in undoped a$\mathrm{Si}: \mathrm{H}$ [29] and a-SiGe:H alloys [25] on the sub-bandgap absorption spectrum were mostly overlooked.

In this paper, we have used an alternative sub-bandgap absorption method in order to measure reliable and accurate sub-bandgap absorption $\alpha(\mathrm{h} v)$ spectrum. Dual beam photoconductivity (DBP) method used extensively in the research of undoped a-Si:H films [29, 30] was improved with optical transmission spectrum simultaneously measured from the back of sample and used, for the first time, to calculate interference fringe free absolute $\alpha(\mathrm{h} v)$ spectrum from 2.5 to $0.6 \mathrm{eV}$ for the undoped a-SiGe:H alloy films both in the annealed and light soaked states. Absolute subbandgap absorption $\alpha(h v)$ spectra of samples in the annealed state calculated from DBP yield and optical transmission spectra using Ritter-Weiser Formula [31] were also compared with those independently measured PDS spectra on the same a-SiGe:H alloy. A perfect agreement was obtained between the $\alpha(\mathrm{h} v)$ spectra measured by PDS and indirectly calculated from DBP and optical transmission in a wider energy region, consistent differences exist only in the sub-bandgap enegies due to differences in the probing nature of PDS and DBP methods. Finally, the improved DBP and SSPC methods were applied to investigate the SWE in a-SiGe:H alloys with varying $\mathrm{Ge}$ content by measuring the changes in the $\alpha$ (hv) spectrum and mobility-lifetime product of majority carriers.

\section{Experimental details}

Hydrogenated amorphous silicon-germanium alloy thin films (a-SiGe:H) of various germanium concentrations used in this study were deposited by using Plasma Enhanced Chemical Vapor Deposition (PECVD) technique at standard $13.56 \mathrm{MHz}$ on glass substrates at Forschungszentrum Julich, Germany using $\mathrm{Si}_{2} \mathrm{H}_{6}+\mathrm{GeH}_{4}+\mathrm{H}_{2}$ gas mixture [32]. The process temperature was $200{ }^{\circ} \mathrm{C}$ and pressure was 700 mTorr. Ge concentration of the samples was changed by changing the $\mathrm{GeH}_{4}$ ratio in total gas mixture between $0 \%(\mathrm{a}-\mathrm{Si}: \mathrm{H})$ and $75 \%$. Corresponding $\mathrm{Ge}$ content of the samples were found to be $0,5,10,20,28,40$ and $62 \%$ for samples deposited with $0,10,20,30,40,50$, and $75 \% \mathrm{GeH}_{4}$ ratio, respectively [33]. SSPC, $\sigma_{\mathrm{ph}}$, measurements in the annealed and light soaked states were measured using 690 and $750 \mathrm{~nm}$ interference filters and RG610 filter, together with a calibrated ENH type white light source. Light soaking procedure is carried out until samples reach to the stabilized steady-state by leaving the film under an intensity of 15 Suns of white light for predetermined time intervals. The temperature during soaking was kept constant at about $40{ }^{\circ} \mathrm{C}$ by using fans and water filters. PDS measurements were carried out at Institute of Photovoltaics of Forschungszentrum Julich, Germany. In this study, DBP yield spectrum, $\mathrm{Y}_{\mathrm{DBP}}$, measured at high and low bias light intensities and simultaneously measured transmission signal from the substrate side of the sample were used to calculate absolute $\alpha(\mathrm{h} v)$ spectrum using a procedure [34] based on the Ritter-Weiser formula [31].

\section{Results and discussions}

\subsection{Annealed state}

Mobility-lifetime, $\eta \mu \tau$, product versus generation rate of majority carriers calculated from the $\sigma_{\mathrm{ph}}$ measurements for a-SiGe:H samples with varying $\mathrm{Ge}$ concentrations from 0 to $62 \%$ are shown in Fig. 1. $\eta \mu \tau$-products exhibit the 


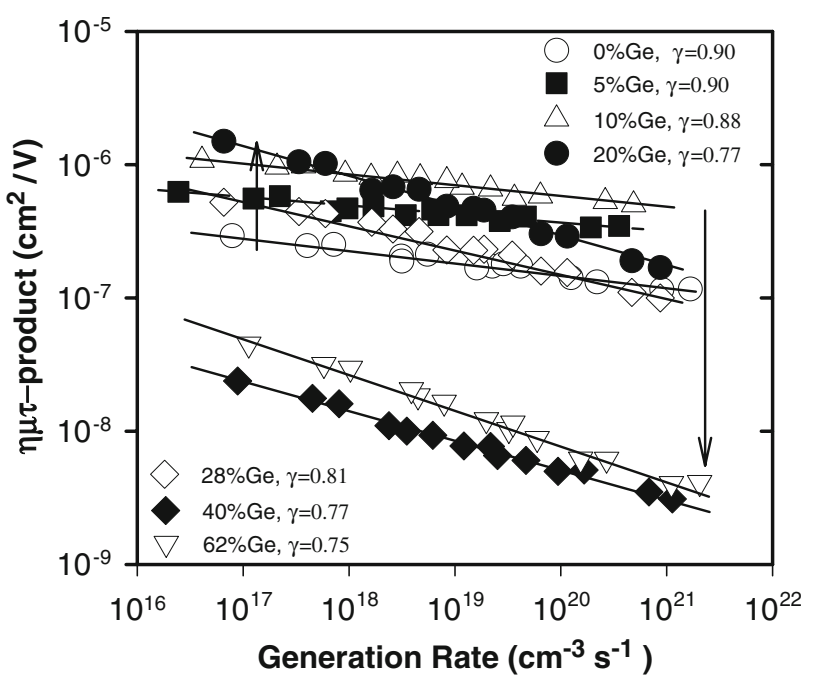

Fig. $1 \eta \mu \tau$-Products versus generation rate of a-SiGe:H alloys with Ge content from 0 to $62 \%$ in the annealed state

expected linear relation as a function of generation rate. Magnitude of $\eta \mu \tau$-products increase as Ge content changes from $0 \%$ (pure a-Si:H) to $10 \%$ and the exponent $\gamma$ stays around 0.90 . Further increase in Ge concentration results in a decrease in photosensitivity and a sharp drop exists for samples with 40 and $62 \%$ Ge concentration. Correspondingly, the exponent $\gamma$ begins to decrease below 0.90 , indicating that there exists a substantial change in the density and nature of native defects states present in the bandgap of the a-SiGe:H alloys as Ge concentration increases above $28 \%$.

The density of these native defect states present in amorphous network is generally characterized by using the sub-bandgap absorption measurements. In this study, DBP method and simultaneously measured transmission spectrum were used to calculate absolute absorption coefficient spectrum. In the inset of Fig. 2, raw DBP yield spectra, $\mathrm{Y}_{\mathrm{DBP}}$, and corresponding transmission signal are shown for a-SiGe:H sample with $20 \%$ Ge concentration. Interference fringes existing in the $Y_{D B P}$ and transmission spectra and peak positions of fringes in both spectra are found at the same energy values, indicating homogeneous absorption of light in the sample. Independently calculated $\alpha(\mathrm{h} v)$ spectra of DBP measurements under high and low generation rates are presented together in Fig. 2. It is clearly seen that both spectra overlap very well above $1.4 \mathrm{eV}$ and deviation exists only in the sub-bandgap energies without any remaining fringes on the spectra. The increase in the $\alpha(\mathrm{h} v)$ spectrum at the lower energy values is due to an increase in the occupation of the defect states in the bandgap by the higher intensity of bias light used in DBP technique, which moves the quasi Fermi levels closer to the extended band edges. An increase in the optical transitions at the lower energies, from the occupied defect states localized in the bandgap

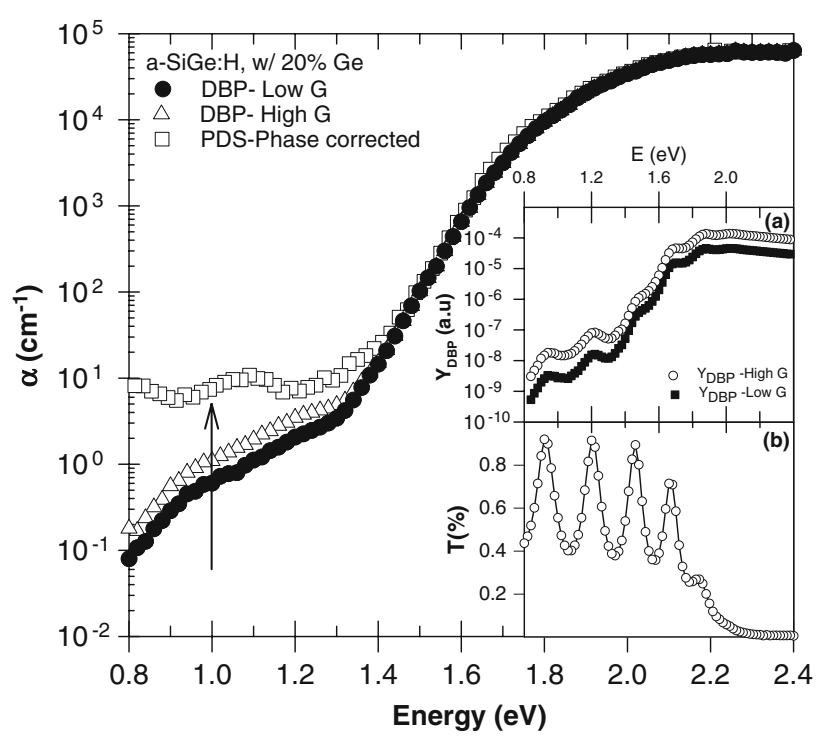

Fig. 2 Independently measured sub-bandgap absorption coefficient spectra of a-SiGe:H alloy thin film with $20 \%$ Ge concentration in the annealed state by PDS and DBP methods. In the insets raw DBP yield spectra measured with high and low bias light intensities and corresponding transmission signal measured from the substrate side of the sample are shown

into the conduction band edge, causes higher optical absorption coefficients only in the sub-bandgap energies as measured by high bias light DBP measurements. To obtain the effects of occupied defect states below dark Fermi level on the optical absorption spectrum, intensity of bias light used in DBP measurement is generally maintained as low as possible. In this study, low generation rate DBP spectrum was used to compare the results of the alloy samples.

In order to compare the accuracy of $\alpha$ (hv) spectrum calculated from DBP measurements, an independent measurement of sub-bandgap absorption spectrum was carried out on the identical sister samples taken from the same substrate using PDS. PDS spectrum of the same sample is shown in Fig. 2 together with those calculated from DBP and transmission measurements which show a perfect overlap at higher energies above $1.4 \mathrm{eV}$. However, a substantial difference between PDS spectrum and that of DBP low generation rate exists in the sub-bandgap energies. In PDS spectrum, a phase correction procedure was applied to remove the effect of absorbing substrate causing a phase shift in raw PDS signal [34]. Similar procedures for subbandgap absorption measurements were also carried out for other a-SiGe:H alloy thin films with different Ge concentration using both methods. It was found that similar $\alpha$ (hv) spectra as shown in Fig. 2 were obtained for the other alloy thin films, where PDS spectrum is always higher than that of low generation rate DBP spectrum.

The results of sub-bandgap absorption spectra of the alloy thin films measured by DBP indicate that the bandgap 
of a-SiGe:H alloy decreases as Ge content increases, as previously reported in literature [1-8, 18]. The bandgap $\mathrm{E}_{04}$ defined for $\alpha(\mathrm{h} v)$ equals to $10^{4} \mathrm{~cm}^{-1}$ is $1.92 \mathrm{eV}$ for $0 \% \mathrm{Ge}$ (a-Si:H) film and decreases to the energy values of 1.90 , $1.86,1.80,1.75,1.58$, and $1.46 \mathrm{eV}$ for $5,10,20,28,40$, and $62 \% \mathrm{Ge}$ content sample, respectively. Correspondingly, the slope of the exponential absorption tail, $\mathrm{E}_{0 \mathrm{v}}$, calculated from the $\alpha(h v)$ spectrum at the energies between 1.4 and $1.7 \mathrm{eV}$ is $54 \pm 2 \mathrm{meV}$ for samples with $\mathrm{Ge}$ content between 0 and $28 \% \mathrm{Ge}$ and increases to $60 \pm 2 \mathrm{meV}$ for 40 and $62 \% \mathrm{Ge}$ samples. The values of $\mathrm{E}_{0 \mathrm{v}}$ indicate that the level of disorder in these alloy samples is in the accepted limit of standart quality electronic thin films.

The effect of Ge concentration on the sub-bandgap absorption measurements in the annealed state is presented in Fig. 3. The absorption coefficient at $1.0 \mathrm{eV}, \alpha(1.0 \mathrm{eV})$, was taken as comparison criteria for the level of the density of defect states present in the samples. In Fig. $3 \mathrm{a}$, the $\alpha$ $(1.0 \mathrm{eV})$ measured using DBP low generation rate decreases as Ge concentration increases from 0 to $10 \%$. The alloy with $10 \%$ Ge content has the lowest $\alpha(1.0 \mathrm{eV})$, indicating the lowest defect density. This result is consistent with the highest $\eta \mu \tau$-products of the $10 \%$ Ge sample shown in Fig. 3b. Further increase in Ge content results in

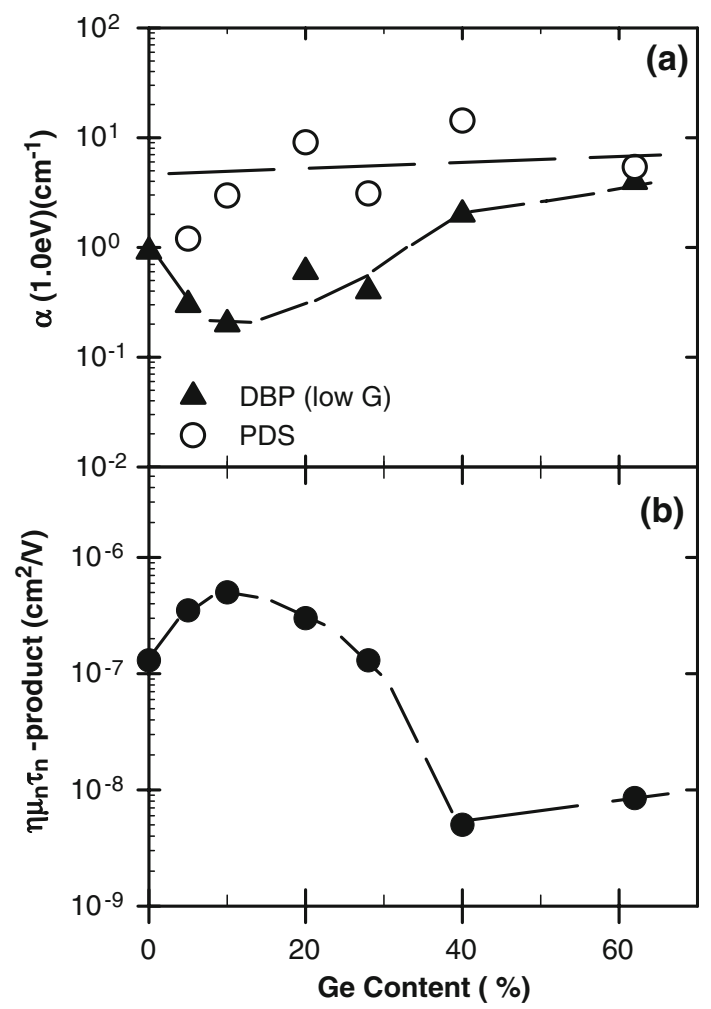

Fig. 3 a Sub-bandgap absorption coefficient at $1.0 \mathrm{eV}$ versus $\mathrm{Ge}$ concentration obtained from PDS and DBP methods, $\mathbf{b} \eta \mu \tau$-products measured at $G=10^{20} \mathrm{~cm}^{-3} \mathrm{~s}^{-1}$ versus Ge content in the annealed state. Lines are guide to eye an increase in the defect states as $\alpha(1.0 \mathrm{eV})$ increases. Correspondingly, it causes more recombination and degrades the $\eta \mu \tau$-products as given in Fig. $3 \mathrm{~b}$. A substantial decrease in $\eta \mu \tau$-products for samples above $28 \%$ Ge concentration points out that there could be an important change in the nature of the defect states in the high Ge content alloys. A good agreement exists between changes in $\alpha(1.0 \mathrm{eV})$ obtained from DBP low bias light and changes in $\eta \mu \tau$-products as $\mathrm{Ge}$ concentration changes. However, such distinct functional dependence of $\alpha$ $(1.0 \mathrm{eV})$ cannot be seen from the PDS measurement as indicated in Fig. 3. These results indicate that both DBP and PDS methods are complementary to one another and can be used to obtain reliable absorption coefficient spectrum above the bandgap energies. However, PDS should be carefully practiced at the lower energies for the investigation of bulk defects states present in the material.

\subsection{Light soaked state}

Light induced degradation known as the SWE [17] is an intrinsic property of hydrogenated amorphous silicon thin films. It was also reported that a-SiGe:H alloys exhibit similar SWE as exposed to sun light $[10,11]$. In the inset of Fig. 4, SSPC versus generation rate measured after different light soaking periods is presented for the a-SiGe:H alloy with $20 \%$ Ge concentration. Magnitude of $\sigma_{\mathrm{ph}}$ decreases more than two orders of magnitude when sample reached to stabilized steady-state and the exponent $\gamma$

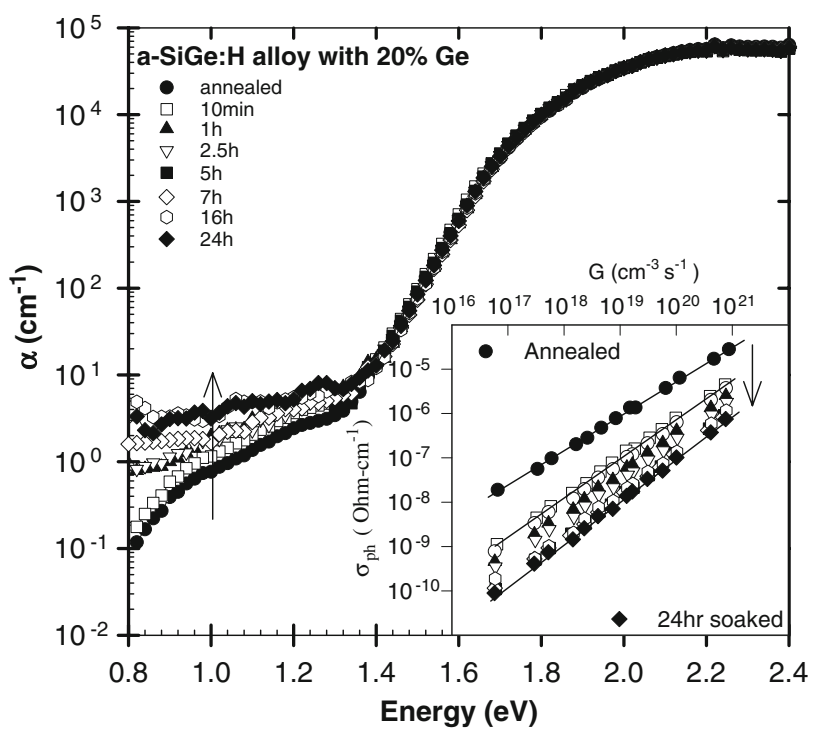

Fig. 4 The changes in the sub-bandgap absorption coefficient spectra with extended light soaking for the a-SiGe:H alloy with $20 \%$ Ge. In the inset corresponding steady-state photoconductivity versus generation rate data is shown for different periods of light soaking 
increases from its annealed state value to 0.85 . For the light soaked state, $\sigma_{\mathrm{ph}}$ versus light soaking time taken at a constant generation rate $\left(G=1 \times 10^{17} \mathrm{~cm}^{-3} \mathrm{~s}^{-1}\right)$ was plotted using the data shown in the inset of Fig. 4. It was found that degradation of $\sigma_{\mathrm{ph}}$ obeys to $t^{-x}$ functional dependence, where $x$ is calculated to be 0.55 , much higher than that generally reported $t^{-1 / 3}$ rule for a-Si:H thin films [35]. Similar degradation kinetics were also obtained for other a-SiGe:H alloy thin films as Ge content changes from 0 to $28 \%$. The value of $x$ is 0.50 for pure a-Si:H $(0 \% \mathrm{Ge})$ and $0.60,0.55$ and 0.30 for a-SiGe:H alloy with 10, 20, and $28 \% \mathrm{Ge}$, respectively. However, degradation becomes less significant for samples with 40 and $62 \%$ Ge concentration, where $x$ is 0.11 and 0.05 , respectively. Similar degradation kinetics of high Ge alloys with $40 \% \mathrm{Ge}$ and above were also reported previously using the ESR measurements [10].

Degradation of $\sigma_{\mathrm{ph}}$ seen in the inset of Fig. 4 is attributed to the creation of light induced defect states in the bandgap of a-SiGe:H alloy, which decrease the majority carrier lifetime through increased recombination kinetics. The changes in the density of defect states after light soaking are commonly characterized by the sub-bandgap absorption techniques. In this study, DBP measurements were extended for the investigation of light soaked state in the alloy samples. In Fig. 4, absolute $\alpha$ (hv) spectra calculated from the low generation rate DBP measurements are shown for the sample with $20 \%$ Ge concentration. The $\alpha(h v)$ values below the exponential absorption edge increases as light soaking period increases as indicated by arrow sign in Fig. 4. Due to large degradation of intrinsic photoconductivity of the samples, ac photoconductivity signal of DBP measurements becomes very weak in the sub-bandgap energies. For this reason, the $\alpha(\mathrm{h} v)$ spectrum in the sub-bandgap energies can be defined by a certain degree of experimental error. Time dependence of $\alpha$ $(1.0 \mathrm{eV})$ is obtained from the data shown in Fig. 4. It was found that $\alpha(1.0 \mathrm{eV})$ versus time obeys to $t^{y}$ functional dependence, where $y$ is equal to 0.25 for $20 \%$ Ge alloy sample. It is indicated that there is no one-to-one correlation between degradation of SSPC and increase in the subbandgap absorption as measured by DBP technique.

Similar experimental results were obtained for the degradation of photoconductivity and the changes in the sub-badgap absorption coefficients for other a-SiGe:H alloy thin films. In order to correlate the degradation of $\sigma_{\mathrm{ph}}$ and the changes in the $\alpha(1.0 \mathrm{eV}),\left[\mu \tau_{\text {soak }} / \mu \tau_{\text {anneal }}\right]^{-1}$ taken at $G=1 \times 10^{17} \mathrm{~cm}^{-3} \mathrm{~s}^{-1}$ and $\left[\alpha(1.0 \mathrm{eV})_{\text {soak }} / \alpha\right.$ $\left.(1.0 \mathrm{eV})_{\text {anneal }}\right]$ versus light soaking time were presented together in Fig. 5. Both parameters, $\left[\mu \tau_{\text {soak }} / \mu \tau_{\text {anneal }}\right]^{-1}$ and $\left[\alpha(1.0 \mathrm{eV})_{\text {soak }} / \alpha(1.0 \mathrm{eV})_{\text {anneal }}\right]$, are equal to unity in the annealed state and increase as light soaking time increases. An approximate solid line in each figure was drawn for a guide to eye, which also points the annealed state values of

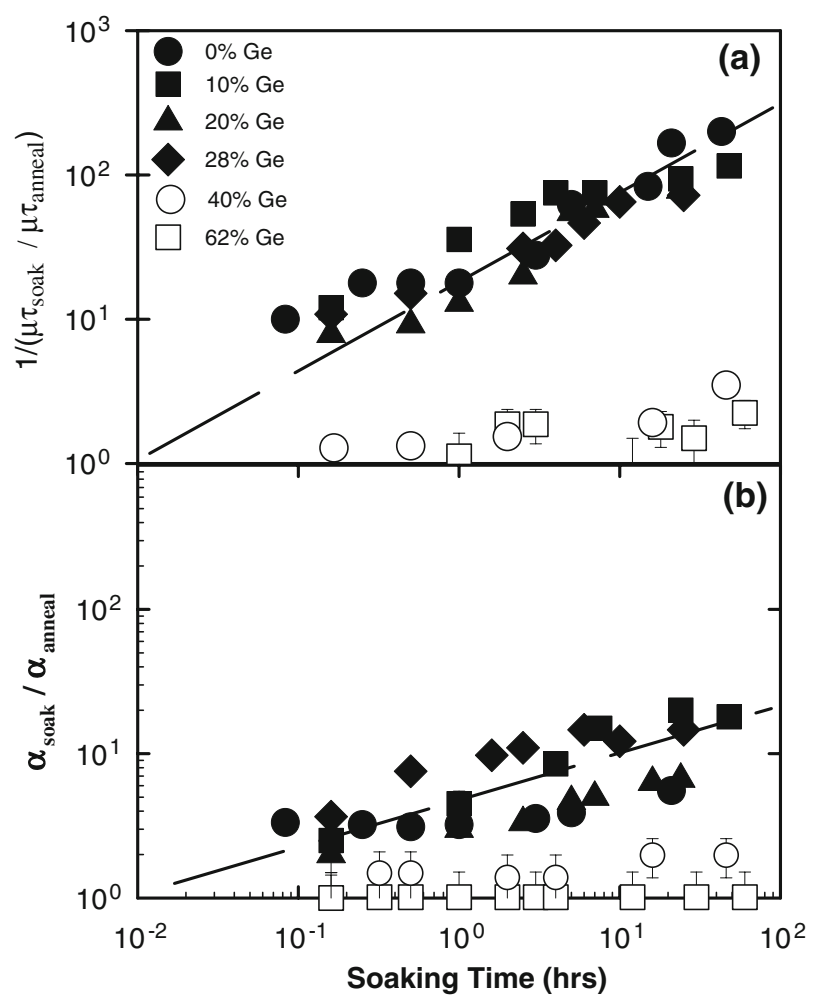

Fig. 5 a $1 /\left(\mu \tau_{\text {soak }} / \mu \tau_{\text {anneal }}\right)$ versus soaking time, $\mathbf{b} \alpha_{\text {soak }} / \alpha_{\text {anneal }}$ versus soaking time for the a-SiGe:H alloys with Ge concentration from 0 to $62 \%$

both parameters. The changes in both parameters are due to creation of new light induced defect states which affect directly both experimental SSPC and sub-bandgap absorption measurements. It is clearly seen that degradation of a-SiGe:H alloys with Ge concentration from $0 \%$ (pure a-Si:H) to $28 \%$ exhibit similar functional dependence on time for both parameters. However, a-SiGe:H alloys with 40 and $62 \%$ Ge concentration show almost insignificant degradation in both parameters. An important difference between these two figures is that the slope of $\left[\mu \tau_{\text {soak }} /\right.$ $\left.\mu \tau_{\text {anneal }}\right]^{-1}$ is approximately two times higher than that of $\left[\alpha(1.0 \mathrm{eV})_{\text {soak }} / \alpha(1.0 \mathrm{eV})_{\text {anneal }}\right]$, indicating that defects causing the degradation of $\eta \mu \tau$-products and increase of sub-bandgap absorption coefficient, $\alpha(1.0 \mathrm{eV})$, are not the same type of defects. Insignificant degradation kinetics of high Ge concentration alloys were also reported in previous investigations $[10,15,36]$. These results indicate that degradation kinetics in a-Si:H and a-SiGe:H alloys with Ge concentration up to $28 \%$ are similar in nature as in agreement with previous reports. However, detailed nature of defect creation in these alloys, especially the ratio of native $\mathrm{Si}$ and Ge related defects and the ratio of light induced creation of $\mathrm{Si}$ and $\mathrm{Ge}$ related defects in a-SiGe:H alloys with different $\mathrm{Ge}$ concentrations, cannot be directly understood from these results alone. 


\section{Conclusions}

The effects of native and light induced defects states in a-SiGe:H alloys with varying Ge concentration on the optoelectronic properties were investigated in the annealed and light soaked states. In the annealed state, $\eta \mu \tau$-products have the highest values for the alloy with $10 \% \mathrm{Ge}$ concentration. It decreases as Ge content increases to $28 \%$. There exists a drastic decrease in $\eta \mu \tau$-products for film with 40 and $62 \%$ Ge concentration. Reliable sub-bandgap absorption coefficient spectrum in a wider energy range is obtained from independently measured DBP and PDS spectra. A perfect overlap is obtained between two measurements at the energies above $1.4 \mathrm{eV}$. However, a substantial difference between the spectra exists at the lower energies, where PDS spectrum is always higher than that of DBP. Functional dependence of defect related $\alpha(1.0 \mathrm{eV})$ obtained from the low generation rate DBP spectrum on Ge concentration indicates that the alloy with $10 \%$ Ge concentration has the lowest density of native defects, consistent with the highest $\eta \mu \tau$-products measured in the annealed state. As Ge content increases above 10\%, $\alpha$ $(1.0 \mathrm{eV})$ increases, meaning that samples become more defective and less photoconductive. Good agreement was found between functional dependence of $\eta \mu \tau$-products and $\alpha(1.0 \mathrm{eV})$ obtained from DBP measurement on Ge concentration. However, it is difficult to make such correlation between $\alpha(1.0 \mathrm{eV})$ obtained from PDS and dependence of $\eta \mu \tau$-products on Ge concentration.

In the light soaked state, all a-SiGe:H alloy thin films with low and moderate Ge concentration show SWE under extended white light illumination as observed in undoped a-Si:H thin films [17]. The alloys with highest Ge concentration show almost insignificant degradation both in SSPC and sub-bandgap absortion spectrum. For the samples up to $28 \%$ Ge concentration, SSPC decreased more than an order of magnitude as samples reached to the stabilized steady-state. Correspondingly, sub-bandgap absorption coefficient spectrum increased only at lower energies. Time dependence of SSPC degradation is almost two times higher than that of increase in $\alpha(1.0 \mathrm{eV})$. However, detailed nature of defect creation in the alloys with different Ge concentrations cannot be directly elucidated from these results alone. Therefore, complete understanding of light induced defect creation in the a-SiGe:H alloys is still missing. Additional investigations on the light induced defect creation using different methods on the same set of samples and experimental conditions are required. Such studies will allow us to make correlations between two distinct types of light induced defects in the alloys as well as in undoped a-Si:H thin films.
Acknowledgments Authors would like to thank Dr. R. Carius for the fruitful discussion.

\section{References}

1. J. Yang, A. Banerjee, S. Guha, Appl. Phys. Lett. 70, 2975 (1997). doi:10.1063/1.118761

2. J. Yang, A. Banerjee, S. Guha, Sol. Energy Mater. Sol. Cells 78, 597 (2003). doi:10.1016/S0927-0248(02)00453-1

3. D. Della Sala, C. Reita, G. Conte, F. Galluzi, G. Grillo, J. Appl. Phys. 67, 814 (1990). doi:10.1063/1.345737

4. F. Finger, W. Fuhs, G. Beck, R. Carius, J. Non-Cryst. Solids 97/ 98, 1015 (1987). doi:10.1016/0022-3093(87)90244-4

5. R.A. Street, C.C. Tsai, M. Stutzmann, J. Kakalios, Philos. Mag. B 56, 289 (1987). doi:10.1080/13642818708221318

6. J. Bullot, M. Galin, M. Gauthier, B. Bourdon, J. Phys. 44, 713 (1983)

7. G. Nakamara, K. Sato, Y. Yukimoto, Sol. Cells 9, 75 (1983). doi: 10.1016/0379-6787(83)90077-7

8. S. Aljishi, Z.E. Smith, S. Wagner, in Amorph silicon \& related mats, ed. by H. Fritzsche (World Scientific, Singapore, 1989), p. 887

9. S. Guha, J.S. Payson, S.C. Argawal, S.R. Ovshinsky, J. NonCryst. Solids 97-98, 1455 (1988)

10. M. Stutzmann, R.A. Street, C.C. Tsai, J.B. Boyce, S.E. Ready, J. Appl. Phys. 66, (1989)

11. C.E. Nebel, H.C. Weller, G.H. Bauer, Mater. Res. Soc. Symp. Proc. 118, 507 (1988)

12. W. Paul, J.H. Chen, E.Z. Liu, A.E. Wetsel, P. Wickboldt, J. NonCryst. Solids 164-166, 1 (1993)

13. S. Guha, J. Yang, S.J. Jones, Y. Chen, D.L. Williamson, Appl. Phys. Lett. 61, 1444 (1992)

14. T. Unold, J.D. Cohen, C.M. Fortmann, Appl. Phys. Lett. 64, 1714 (1994)

15. G. Schumm, C.D. Abel, G.H. Bauer, Mater. Res. Soc. Symp. Proc. 258, 505 (1992)

16. J.D. Cohen, in Properties of amorphous silicon and its alloys, EMIS, datareview series no. 19, ed. by T. Searle (INSPEC, London, 1998), p. 180

17. D.L. Staebler, C.R. Wronski, Appl. Phys. Lett. 31, 292 (1977)

18. K.D. Mckenzie, J.R. Eggert, D.J. Leopold, Y.M. Li, S. Lin, W. Paul, Phys. Rev. B 31, 2198 (1985)

19. L. Chen, J. Tauc, J.K. Lee, E.A. Shiff, Phys. Rev. B 43, 11694 (1991)

20. K.C. Palinginis, J.D. Cohen, J.C. Yang, S. Guha, J. Non-Cryst. Solids 266-269, 665 (2000)

21. C.E. Michelson, A.V. Gelatos, J.D. Cohen, Appl. Phys. Lett. 47, 412 (1985)

22. N.W.W. Wang, Ph.D. Thesis, Princeton University, USA, 1994

23. N.W. Wang, P.A. Morin, V. Chu, S. Wagner, Mater. Res. Soc. Symp. Proc. 258, 589 (1992)

24. A.E.W. Tai, Ph.D. Theis, Harvard University, USA, 1994

25. C.C. Chen, F. Zhong, J.D. Cohen, J. Yang, S. Guha, Phys. Rev. B57, R4210 (1998)

26. J.D. Cohen, D. Kwon, J. Non-Cryst. Solids 227-230, 348 (1998)

27. R. Carius, H. Steibeg, F. Siebke, J. Folsch, J. Non-Cryst. Solids 227-230, 432 (1998)

28. X. Xu, J. Yang, S. Guha, Appl. Phys. Lett. 62, 1399 (1993)

29. M. Gunes, C.R. Wronski, J. Appl. Phys. 81, 3526 (1997)

30. C.R. Wronski, B. Abeles, T. Tiedje, G. Cody, Solid State Commun. 44, 1423 (1982)

31. D. Ritter, K. Weiser, Optics Commun. 57, 336 (1986) 
32. D. Lundszied, J.Fölsch, F. Finger, W. Wagner, 2nd World Conference and Exhibition on Photovoltaic Solar Energy Conversion, (1998) p. 948

33. D. Lundszien, Ph.D. Thesis, Berichte des Forschungszentrums Jülich, 3840; ISSN 0944-2952 (2001)
34. Carius R Private Communication, Research Center Julich, Germany

35. M. Stutzmann, W.B. Jackson, C.C. Tsai, Phys. Rev. B 32, 23 (1985)

36. J. Kolodzey, S. Aljishi, Z.E. Smith, V. Chu, R. Schwarz, S. Wagner, Mater. Res. Soc. Symp. Proc. 70, 237 (1986) 